\title{
Exploring the R CrA environment with SPHERE
}

\section{Discovery of a new stellar companion $\star, \star \star$}

\author{
D. Mesa ${ }^{1,2}$, M. Bonnefoy ${ }^{3}$, R. Gratton ${ }^{1}$, G. Van Der Plas ${ }^{3}$, V. D’Orazi ${ }^{1}$, E. Sissa ${ }^{1}$, A. Zurlo ${ }^{4,5,6}$, E. Rigliaco ${ }^{1}$, \\ T. Schmidt ${ }^{7,8}$, M. Langlois ${ }^{9,6}$, A. Vigan ${ }^{6}$, M. G. Ubeira Gabellini ${ }^{10}$, S. Desidera ${ }^{1}$, S. Antoniucci ${ }^{11}$, M. Barbieri ${ }^{2}$, \\ M. Benisty ${ }^{12,3}$, A. Boccaletti ${ }^{8}$, R. Claudi ${ }^{1}$, D. Fedele ${ }^{13}$, D. Gasparri ${ }^{2}$, T. Henning ${ }^{14}$, M. Kasper ${ }^{15}$, A.-M. Lagrange ${ }^{3}$, \\ C. Lazzoni $^{1}$, G. Lodato ${ }^{10}$, A.-L. Maire ${ }^{14}$, C. F. Manara ${ }^{15}$, M. Meyer ${ }^{16}$, M. Reggiani ${ }^{17}$, M. Samland ${ }^{14}$, \\ M. Van den Ancker ${ }^{15}$, G. Chauvin ${ }^{3}$, A. Cheetham ${ }^{18}$, M. Feldt ${ }^{14}$, E. Hugot ${ }^{6}$, M. Janson ${ }^{14,19}$, R. Ligi ${ }^{20}$, \\ O. Möller-Nilsson ${ }^{14}$, C. Petit ${ }^{21}$, E. L. Rickman ${ }^{18}$, F. Rigal ${ }^{22}$, and F. Wildi ${ }^{18}$
}

(Affiliations can be found after the references)

Received 19 November 2018 / Accepted 6 February 2019

\begin{abstract}
Aims. R Coronae Australis ( $\mathrm{R} \mathrm{CrA}$ ) is the brightest star of the Coronet nebula of the Corona Australis (CrA) star forming region. This star is very red in color, probably due to dust absorption, and is strongly variable. High-contrast instruments allow for an unprecedented direct exploration of the immediate circumstellar environment of this star.

Methods. We observed R CrA with the near-infrared (NIR) channels (IFS and IRDIS) of SPHERE at the Very Large Telescope (VLT). In this paper, we used four different epochs, three of which are from open time observations while one is from SPHERE guaranteed time. The data were reduced using the data reduction and handling pipeline and the SPHERE Data Center. We implemented custom IDL routines on the reduced data with the aim to subtract the speckle halo. We have also obtained pupil-tracking $H$-band $(1.45-1.85 \mu \mathrm{m})$ observations with the VLT/SINFONI NIR medium-resolution $(R \sim 3000)$ spectrograph.

Results. A companion was found at a separation of $0.156^{\prime \prime}$ from the star in the first epoch and increasing to 0.184" in the final epoch. Furthermore, several extended structures were found around the star, the most noteworthy of which is a very bright jet-like structure northeast from the star. The astrometric measurements of the companion in the four epochs confirm that it is gravitationally bound to the star. The SPHERE photometry and SINFONI spectrum, once corrected for extinction, point toward a spectral type object that is early $M$ with a mass between 0.3 and $0.55 M_{\odot}$. The astrometric analyis provides constraints on the orbit paramenters: $e \sim 0.4$, semimajor axis at 27-28 au, inclination of $\sim 70^{\circ}$, and a period larger than $30 \mathrm{yr}$. We were also able to put constraints of few $M_{\text {Jup }}$ on the mass of possible other companions down to separations of few tens of au.
\end{abstract}

Key words. instrumentation: spectrographs - methods: data analysis - techniques: imaging spectroscopy - stars: pre-main sequence stars: individual: $\mathrm{R} \mathrm{CrA}$

\section{Introduction}

Nowadays young stars surrounded by a protoplanetary disk are considered as the primary environment in which to study the formation of giant exoplanets (see, e.g., Chen et al. 2012; Marshall et al. 2014). In addition, it is clear that giant planets at large separations are more frequently found around intermediate mass stars than around solar-mass stars (see, e.g., Johnson et al. 2010; Bowler 2016). Herbig AeBe (HAeBe) stars (Herbig 1960; Hillenbrand et al. 1992) are young $(<10 \mathrm{Myr})$, intermediate mass (1.5-8 $\left.M_{\odot}\right)$ and typically with $A, B, F$ spectral types. These stars are characterized by a strong infrared excess (The 1994) owing to the presence of a circumstellar protoplanetary disk. They are also experiencing accretion processes and their spectral line profiles are very complex. These characteristics make these objects very valuable in the field of extrasolar planets given that they

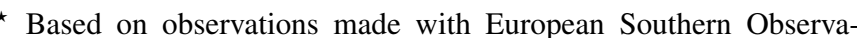
tory (ESO) telescopes at Paranal Observatory in Chile, under programs ID 095.C-0787(A), 097.C-0591(A), 1100.C-0481(H), 0101.C-0350(A) and 2101.C-5048(A).

$\star \star$ The SPHERE and SINFONI images are only available at the CDS via anonymous ftp to cdsarc.u-strasbg. fr $(130.79 .128 .5)$ or via http://cdsarc.u-strasbg.fr/viz-bin/qcat?J/A+A/624/A4
}

allow the exploration of the very first stages of the formation of planetary systems.

The HAeBe star R Coronae Australis (R CrA - HIP 93449) is the brightest star in the very young, compact ( $\sim 1 \mathrm{pc}$ in diameter), and obscured Coronet protostar cluster (Taylor \& Storey 1984). This cluster is characterized by a very high and spatially variable extinction with $A_{V}$ up to $45 \mathrm{mag}$ (Neuhäuser \& Forbrich 2008). This nebula is located toward the center of the Corona Australis (CrA) molecular clouds complex (Graham 1992), one of the nearest star forming region with a distance of $\sim 130 \mathrm{pc}$ (Neuhäuser \& Forbrich 2008). More recently, Dzib et al. (2018), using the mean trigonometric parallax obtained from Gaia Data Release 2 (Gaia Collaboration 2018), estimated a distance of $154 \pm 4 \mathrm{pc}$. The presence of emission line objects was also signaled in the vicinity of R CrA (Graham 1993). The CrA region was explored through deep infrared imaging by Wilking et al. (1997), who identified hundreds of point-like sources. Moreover, these authors gave particular relevance to the study of the region of the Coronet nebula where they identified extensive reflective nebulae, dust-free cavities, and Herbig-Haro objects. More recently, Meyer \& Wilking (2009) obtained the infrared spectra for a magnitude limited sample of stars of the CrA region allowing their characterization. 
The spectral type of R CrA is largely debated and varies from F5 (e.g., Hillenbrand et al. 1992) and A5 (e.g., Chen et al. 1997), to B5III peculiar (Gray et al. 2006), and finally B8 (e.g., Bibo et al. 1992). Other physical charactistics of the star are discussed in more detail in Sect. 3. It is in a very early evolutionary phase (Malfait et al. 1998) with an age of $1_{-0.5}^{+1} \mathrm{Myr}$ (Sicilia-Aguilar et al. 2011) and still embedded in its dust envelope whose emission dominates its spectral energy distribution (SED) from mid-infrared to millimeter wavelengths (Kraus et al. 2009). The mass of the envelope was estimated to be $\sim 10 M_{\odot}$ by Natta et al. (1993) with an outer radius of 0.007 pc corresponding to $\sim 1450$ au. Clark et al. (2000) found a high degree of linear $(\sim 8 \%)$ and circular $(\sim 5 \%)$ polarization as a hint of scattering from dust grains. The polarization map in $V$ band also indicates the presence of an extended ( $\sim 10^{\prime \prime}$ corresponding to more than $1500 \mathrm{au}$ ) disk-like structure with a roughly north-south orientation (Ward-Thompson et al. 1985). A similar orientation was found by Kraus et al. (2009) using interferometry in the nearinfrared (NIR) with VLTI/AMBER; these authors also found asymmetries in the brightness distribution and obtained a disk inclination of $\sim 35^{\circ}$.

Moreover, Takami et al. (2003) proposed the presence of a stellar companion and of an outflow based on their spectroastrometric observations. They estimated for the binary a separation of $\sim 8 \mathrm{au}$ and a period of $\sim 24 \mathrm{yr}$. The presence of a stellar companion was also proposed by Forbrich et al. (2006) to explain the X-ray spectrum of R CrA based on the presence of a strong X-ray emission that is not expected for HAeBe stars.

In this paper we present the SPHERE (Beuzit et al. 2019) view of R CrA and its closeby environment. The paper is organized as follows: In Sect. 2 we describe the observations and the data reduction, in Sect. 3 we discuss the characteristics of R CrA, and in Sect. 4 we report our results, which are then discussed in Sect. 5. Finally, in Sect. 6 we present our conclusions.

\section{Observations and data reduction}

\subsection{SPHERE data}

R CrA was observed with SPHERE, the extreme adaptive optics instrument operating at the ESO Very Large Telescope (VLT) in four epochs. In Table 1 we list all the SPHERE observations used in the present analysis. All these observations were obtained in coronographic mode to be able to suppress the stellar light. The star was observed in a first epoch in the night of 2015 June 10 as part of the open time program 095.C-0787(A) (P.I. G. Van der Plas) aimed to analyze disks of HAeBe stars. The observations were made in IRDIFS_EXT mode that is with IFS (Claudi et al. 2008) operating between $Y$ and $H$ spectral bands $(0.95-1.65 \mu \mathrm{m})$ and IRDIS (Dohlen et al. 2008) operating in dual band configuration with the $K 1-K 2$ filters $(K 1=2.110 \mu \mathrm{m}$ and $K 2=2.251 \mu \mathrm{m}$; Vigan et al. 2010). Moreover, they were taken in field-stabilized mode and in bad weather conditions (see Table 1 for more informations), limiting the contrast that can be reached to a value of $\sim 4 \times 10^{-4}$ at $0.4^{\prime \prime}$.

A second epoch was obtained during the night of 2016 August 10 for the open time program 097.C-0591(A) (P.I. T Schmidt). In this case, the observations were made with IFS operating in $Y$ and $J$ spectral bands (between 0.95 and $1.35 \mu \mathrm{m}$ ) and IRDIS operating with the $\mathrm{H}$ broadband filter (central wavelength $1.625 \mu \mathrm{m}$ with a width of $0.290 \mu \mathrm{m}$ ) instead of the standard $H 2-H 3$ dual band filter. This was a short observation with a total field rotation of $24.7^{\circ}$ and it was taken in weather conditions comparable to those from the first observing epoch.
A third epoch was obtained on 2018 June 19 in the framework of the SHINE (SpHere INfrared survey for Exoplanets; Chauvin et al. 2017) guaranteed time observations (GTO). Like for the first epoch, these observations were made in IRDIFS_EXT mode. In this case the weather conditions were much better than in the previous epochs (Table 1) and the observations were performed in pupil stabilized mode with a total rotation of $71.7^{\circ}$ allowing the implementation of highcontrast imaging methods such as angular differential imaging (ADI; Marois et al. 2006a) and spectral differential imaging (SDI; Racine et al. 1999). This allowed us to reach a much better contrast of $6.7 \times 10^{-6}$ at $0.4^{\prime \prime}$.

Finally, we obtained data in a fourth epoch from the open time program 0101.C-0350(A) (P.I. M.G. Ubeira Gabellini). Also in this case the observations were done with SPHERE operating in IRDIFS_EXT mode and in conditions very similar to the third epoch. The total rotation was $\sim 90^{\circ}$ in this case. For all the epochs, in addition to the coronagraphic observations, we also performed observations with satellite spots symmetric with respect to the central star with the aim to better define the position of the star behind the coronagraph using the method first proposed by Sivaramakrishnan \& Oppenheimer (2006) and Marois et al. (2006b). The use of these spots in the context of the SPHERE observations is discussed in Langlois et al. (2013) and Mesa et al. (2015). Furthermore, observations of the star outside the coronagraph were taken to perform the photometric calibration of the observations. These were observed with an appropriate neutral density filter to avoid saturation of the point spread function (PSF). However, this type of calibration images was not taken in the case of the 2016 August 10 observations, therefore it was not possible to define the contrast of the image and they were only used for astrometric measures using a synthetic Gaussian PSF created for this purpose.

The GTO observations were reduced using the SPHERE data center (Delorme et al. 2017) applying the appropriate calibrations following the data reduction and handling (DRH; Pavlov et al. 2008) pipeline. For the open time observations, instead, we applied directly the DRH pipeline and IDL custom routines for IFS without the intermediation of the SPHERE data center. The required calibration in the case of IRDIS are aimed at the creation of the master dark and master flat-field frames and at the definition of the star center. The steps for the IFS data reduction, instead, include the dark and flat-field correction, definition of the position of each spectra on the detector, wavelength calibration, and application of the instrumental flat. On the reduced data, we then performed algorithms such as principal components analysis (PCA; Soummer et al. 2012) and template locally optimized combination of images (TLOCI; Marois et al. 2014) with the aim to implement speckle subtraction methods such as ADI and SDI. To this aim we used both the IDL procedures described in Mesa et al. (2015) and Zurlo et al. (2014) and the consortium pipeline application called SpeCal (Galicher et al. 2018).

\subsection{SINFONI data}

In this work we also made use of observations of the R CrA system obtained with the AO-fed integral field spectrograph SINFONI (Eisenhauer et al. 2003; Bonnet et al. 2004) during the night of 2018 September 11 (2101.C-5048(A); P.I.: D. Mesa). The spatial sampling was $0.0125^{\prime \prime} \mathrm{pixel}^{-1} \times 0.025^{\prime \prime} \mathrm{pixel}^{-1}$ for a total field of view (FOV) of $0.8^{\prime \prime} \times 0.8^{\prime \prime}$. We observed the target with $H$-band grating operating between $1.45 \mu \mathrm{m}$ and $1.85 \mu \mathrm{m}$ with a resolution $R \sim 3000$. Given the brightness of the star, 
D. Mesa et al.: Exploring R CrA with SPHERE

Table 1. List and main characteristics of the SPHERE observations of R CrA used for this work.

\begin{tabular}{lcccccccc}
\hline \hline Date & Obs. mode & field/pupil & DIMM seeing & $\tau_{0}$ & Wind speed & Rotation & DIT & Total exposure \\
\hline $2015-06-10$ & IRDIFS_EXT & field & $1.35^{\prime \prime}$ & $2.2 \mathrm{~ms}$ & $10.2 \mathrm{~m} \mathrm{~s}^{-1}$ & none & $16 \mathrm{~s}$ & $1680 \mathrm{~s}$ \\
$2016-08-10$ & YJ+BB_H & pupil & $1.37^{\prime \prime}$ & $2.9 \mathrm{~ms}$ & $12.5 \mathrm{~m} \mathrm{~s}^{-1}$ & $24.7^{\circ}$ & $64 \mathrm{~s}$ & $1920 \mathrm{~s}$ \\
$2018-06-19$ & IRDIFS_EXT & pupil & $0.55^{\prime \prime}$ & $6.5 \mathrm{~ms}$ & $7.1 \mathrm{~m} \mathrm{~s}^{-1}$ & $71.7^{\circ}$ & $96 \mathrm{~s}$ & $4608 \mathrm{~s}$ \\
$2018-08-16$ & IRDIFS_EXT & pupil & $0.76^{\prime \prime}$ & $4.6 \mathrm{~ms}$ & $7.8 \mathrm{~m} \mathrm{~s}^{-1}$ & $90.5^{\circ}$ & $12 \mathrm{~s}$ & $5760 \mathrm{~s}$ \\
\hline
\end{tabular}

to avoid saturation we adopted the minimum DIT of $0.83 \mathrm{~s}$. We took 30 datacubes each composed by 40 frames for a total exposure time of $996 \mathrm{~s}$. Moreover, to be able to implement highcontrast imaging techniques like ADI, we observed the target using the pupil tracking mode for a total rotation of the FOV of $\sim 95^{\circ}$.

The data were reduced using the version 3.1.1 of the SINFONI pipeline ${ }^{1}$. The science data were corrected for bad and nonlinear pixels and for distortion. The final calibrated datacubes were then reconstructed from the associated wavelength calibration. On the final calibrated datacubes we then corrected the wavelength dependent drift due to the atmospheric refraction following the same procedure described in Meshkat et al. (2015) and Hoeijmakers et al. (2018). We then subtracted the stellar halo at each of the 2120 wavelengths applying both the classical ADI and PCA techniques. For the SINFONI data, we did not use the SDI technique because, while it could help in gaining in $\mathrm{S} / \mathrm{N}$, on the other hand it could introduce deformations in the object spectrum. Given that the main aim of the SINFONI data is a better classification of the companion object through its spectral lines so that the use of the SDI could lead to a biased result. To derive the correct parallactic angle to be associated with each datacube of our dataset we took the mean of the values of the parallactic angle at the beginning and at the end of each exposure that are given in the header associated with the datacube. These values were then corrected by the value of $304^{\circ}$ to properly orient them with north up and east to the right (see Meshkat et al. 2015 for the details of view orientation of SINFONI pupil-tracking mode).

\section{Stellar parameters}

In this Section we discuss the stellar parameters that are important for our analysis such as the distance, luminosity, and variability. The parallax of the star as measured by Gaia in Data Release 2 (Gaia Collaboration 2018) is $10.53 \pm 0.70$ mas corresponding to a distance of $94.9_{-5.9}^{+6.7} \mathrm{pc}$ that is far from the previous estimated distance of the CrA region $(d \sim 130 \mathrm{pc}$; de Zeeuw et al. 1999). Then, when looking for other objects in the CrA region in the Gaia archive we found lower values for the parallax. To perform this research we selected from the Gaia archive objects with angular separation lower than 10 arcmin from RCrA and a value of the parallax larger than 5 mas. Moreover, we selected all the stars with a proper motion in right


nation smaller than -25 mas $\mathrm{yr}^{-1}$ with the aim to retain only stars with a proper motion similar to that of $\mathrm{R} \mathrm{CrA}$. In this way we selected 15 objects and we verified, through kinematics and literature searches, for each of these sources that it was part of the $\mathrm{CrA}$ region. The median value of the parallax for these objects is $6.54 \pm 0.33$ mas corresponding to a distance of $152.9_{7.3}^{+8.1} \mathrm{pc}$.

\footnotetext{
1 http://www . eso.org/sci/software/pipelines/sinfoni/ sinfoni-pipe-recipes.html
}

Given that the membership of R CrA to the CrA region is well established, the Gaia value for R CrA is probably plagued by an error possibly due to the fact that the star is deeply embedded in its dust envelope. For this reason we decided to assume, for the rest of this work, the distance obtained from the median of the other objects of the CrA region. It is worth to note that the adopted distance for R CrA is very similar to that of the Corona Australis region derived by Dzib et al. (2018).

Another important characteristic of this star is its higher luminosity in the NIR $(J=6.94 ; H=4.95 ; K=3.46$; Ducati 2002; Cutri et al. 2003) than at optical wavelengths $(V=$ 11.92; Koen et al. 2010). However it is also known that this star is strongly variable. So far the only published homogeneous photometric survey of R CrA was carried out at the visible wavelengths exploiting the Maidanak Observatory in Uzbekistan starting from 1983, and was published by Herbst \& Shevchenko (1999). An analysis of these data using a generalized LombScargle periodogram (GLSP; Zechmeister \& Kürster 2009) shows several significative peaks, with separations in frequency corresponding to one year $(0.00276 \mathrm{dex})$. The two highest power frequency peaks are those of 55.7 days and 65.9 days and cannot be due to the data sampling. Furthermore, Percy et al. (2010) studied the photometric stability of R CrA exploiting a $100 \mathrm{yr}$ long visual data sequence provided by the American Association of Variable Star Observers (AAVSO). These authors found a period of 66 days, stable in time but slightly variable in amplitude. The amplitude of this variability was larger than 4 magnitudes at visible wavelengths.

Finally, a fundamental parameter to be analyzed is the extinction in the direction of R CrA, which can strongly affect the extracted spectrum of the star and its companion (see Sect. 4). Bibo et al. (1992) found a value of $E(B-V)=0.99$ and a value of $R_{V}=4.7$ corresponding to an absorption of $A_{V}=4.65$. More recently, Garcia Lopez et al. (2006) found a lower value of $A_{V}=1.40$ while Lazareff et al. (2017) found a value of $A_{V}=3.31$ assuming a value of 3 for $R_{V}$. However, these values were determined for a wide region around the star while the extinction is strongly variable in the Coronet region. For this reason we developed a method to estimate the extinction in the direction of the companion, which is described in Sect. 5.1.

\section{Results}

\subsection{Companion}

A companion is found in the final images of all the epochs of observations. In Fig. 1 we show the final images that we obtained from the 2018 June 19 data that, as explained in Sect. 2, allowed us to obtain much deeper images. The scales used in these images were chosen to better visualize the companion; however the presence of extended structures, which we discuss in much greater detail in Sect. 4.2, is apparent. The companion is less visible in the data taken on 2016 August 10, maybe due to the shorter wavelength setup chosen for this observation 


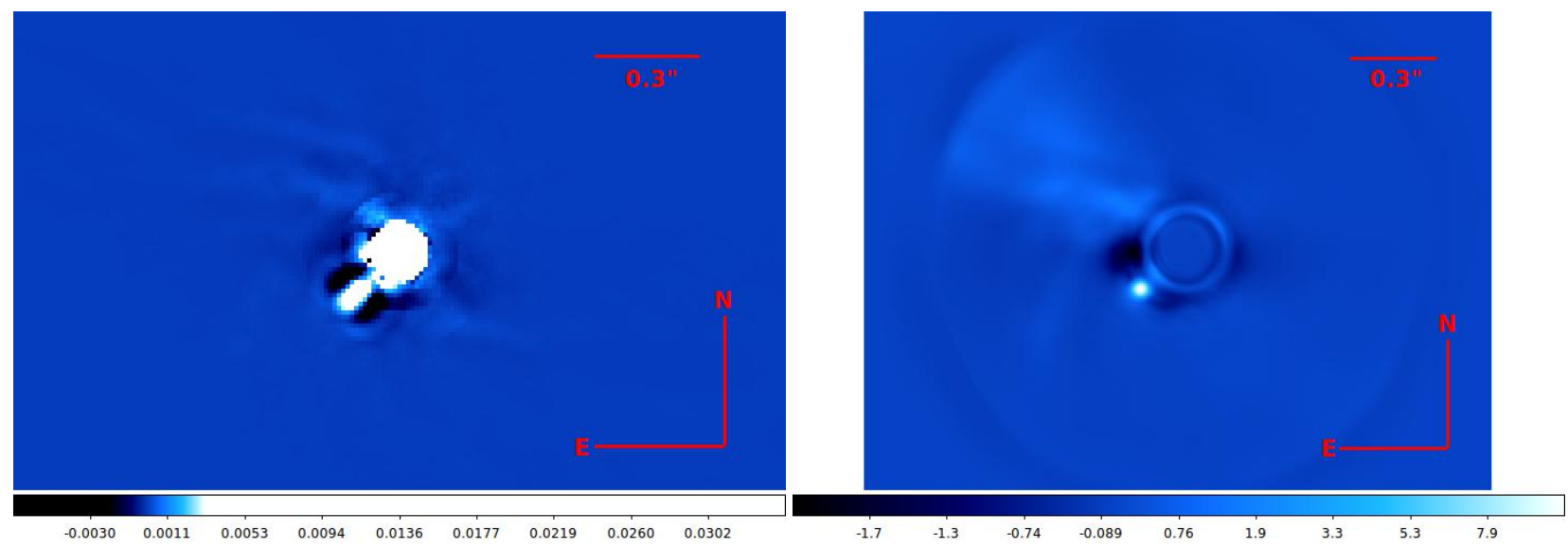

Fig. 1. Final images for R CrA system obtained from the best SPHERE dataset taken on 2018 June 19 (chosen because it is the best quality observation epoch). Both of these are obtained from a median of the images at all the wavelengths covered by the two instruments. Left panel: final image obtained with IRDIS. Right panel: final images obtained with IFS. Both images have flux scales in such a way to make the presence of the companion clear. In both images there are however hints of the presence of the jet-like structure described in Sect. 4.

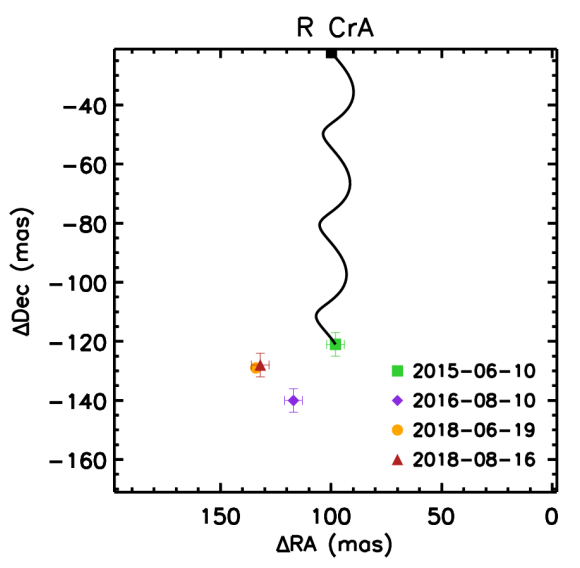

Fig. 2. Relative astrometric position of R CrA B with respect to the host star at four different epochs. The black line represents the path the object would have followed in the three years between the first and fourth epochs. The black square corresponds to the expected position for the companion at the epoch of the third observation if it were a stationary background object.

coupled with a very red spectrum of the source as we show in Sect. 4.1.

In Table 2 we list the relative astrometric values for the companion, while in Fig. 2 we show the relative positions of the companion with respect to the star; these positions are compared to the position that the star would have had if it were a stationary background object, demonstrating that it is actually comoving with the star. We interpret the variations in the companion separation and position angle as evidence of its orbital motion. To further confirm this, we have to consider that the scatter in velocity for CrA members is on the order of $1-2 \mathrm{~km} \mathrm{~s}^{-1}$, while the scatter in velocity between the companion and the central star is between 5 and $10 \mathrm{~km} \mathrm{~s}^{-1}$. This is an other hint that we are actually observing the orbital motion of the companion around the central star.

Two main problems affect the extraction of the photometry. Both of these are linked to the characteristics of the star itself. The first is due to the large difference in magnitude according to the considered spectral band. Given the high brightness of the star in the $H$ and $K$ bands, we had to use the strongest neutral density filter (ND3.5) for the observation of the off-axis PSF to avoid saturation of the star (see Sect. 2). In this way, however, the star was no longer visible in the IFS $Y$ - and $J$-band channels. This of course does not allow a proper flux calibration at these wavelengths. Moreover, the star is strongly variable and for this reason it is difficult to define the absolute magnitude of the companion starting from the contrast (when possible to retrieve). To overcome these problems and to obtain a reliable spectrum of the companion, we then compared the PSF of $\mathrm{R} \mathrm{CrA}$ at each wavelength obtained from the observation of 2018 June 19, when available, to the PSFs of other stars taken during the same night in similar conditions. They are HIP 63847 and HIP 70833, which are not known as variable stars. The results are similar for both stars and they allow us to define a value for $H$ of $6.95 \pm 0.29 \mathrm{mag}$ and for $K$ of $4.63 \pm 0.32 \mathrm{mag}$ for $\mathrm{R} \mathrm{CrA}$ at the epoch of our observation. Also, it was possible to put upper limits to the $Y$ and $J$ magnitudes: $Y>10.92 \mathrm{mag}$ and $J>8.36 \mathrm{mag}$. The comparison of these values with those given in literature (see Sect. 3) puts our observation at (or very near to) the minimum of the star brightness variability. Given the comparable results obtained previously we used as reference the PSF of only HIP 63847 to be able to extract the spectrum in flux for R CrA B shown in Fig. 7. As a first step we calculated the spectrum, in contrast, with respect to the reference star introducing a negative simulated planet at the companion position and ran our speckle subtraction procedure adjusting its flux in such a way as to minimize the standard deviation in a small region around the companion position. We applied this procedure using an ADI and a PCA-based data reduction and we obtained comparable results. The contrast spectrum was then converted to flux by multiplying it by a flux-calibrated BT-NEXTGEN (Allard et al. 2012) synthetic spectrum for HIP 63847 adopting $T_{\text {eff }}=5300 \mathrm{~K}$, $\log g=-0.5$ and $[M / H]=-0.5$ that gives the best fit to the SED of the star according to the VOSA tool (Bayo et al. 2008).

\subsection{Jet-like structure and circumstellar environment}

The environment around $\mathrm{R} \mathrm{CrA}$ is very rich of different extended structures as shown in Fig. 3 in which we represent the final images obtained with IRDIS and IFS with a scale thought to highlight the presence of extended structures. The most striking of these is the jet-like structure northeast from the star that is tagged with 1 in the IFS image (right panel of Fig. 3). The same structure is also clearly visible in the IRDIS image (left panel of 


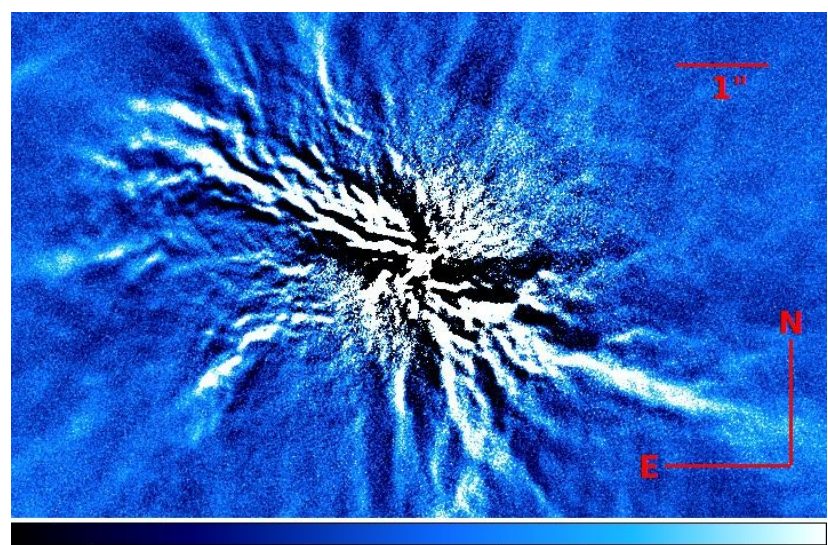

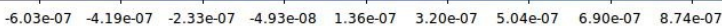

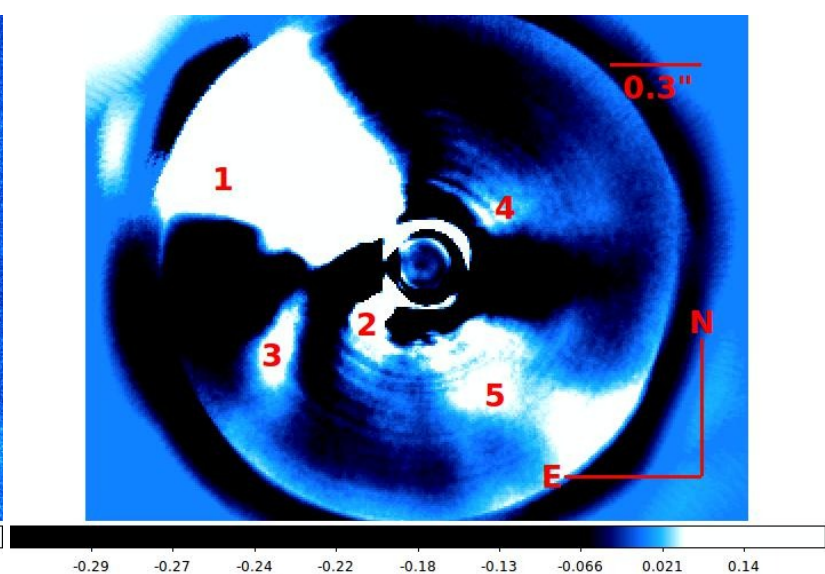

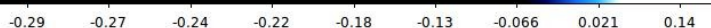

Fig. 3. Final images obtained for IRDIS using SpeCal TLOCI (left panel) and IFS using PCA with 10 principal components (right panel) scaled to enlighten the presence of extended structures. In the IFS image we tag with 1 a jet-like structure, with 2 the position of the companion, with 3 and 4 two faint structures that could be part of the disk, and with 5 a fainter counter-jet.

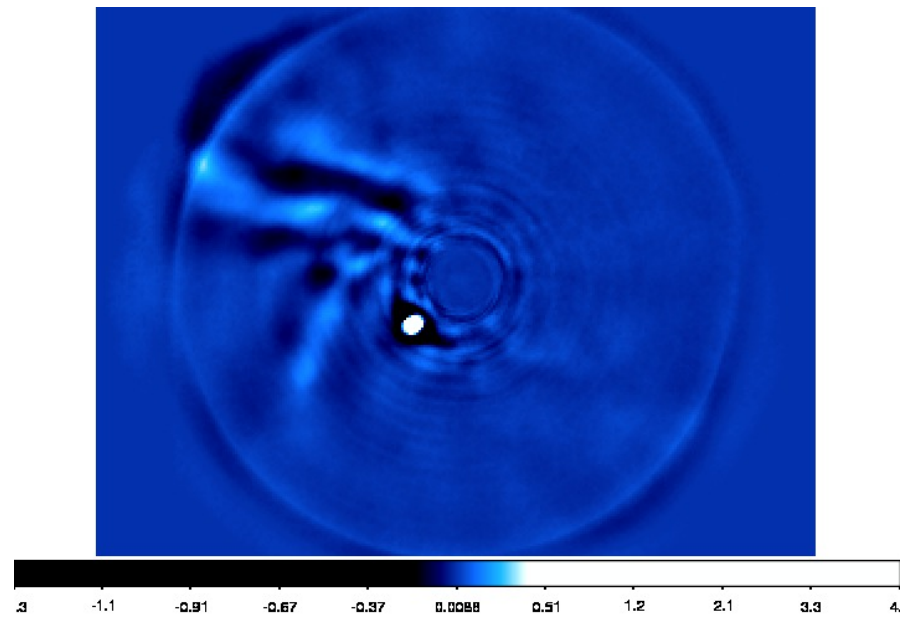

Fig. 4. Final IFS image after the subtraction of PCA 100 principal components aimed to highlight the presence of different substructure into the jet-like structure northeast from R CrA. Scale and orientation are the same as those on the right panel of Fig. 3.

Fig. 3) up to a separation of $3.5^{\prime \prime}$ corresponding to a projected separation of more than $535 \mathrm{au}$. The position angle of median axis of the jet-like structure, calculated using the IFS image, is of $57.8 \pm 8.4^{\circ}$. The IRDIS image allows us to define an aperture of $\pm 14^{\circ}$ at the maximum separation at which it is visible. However, from the IFS image obtained subtracting 100 principal PCA components shown in Fig. 4, it is clear that the jet-like structure consists of at least two separated structures with position angles of $66.1^{\circ}$ and $49.4^{\circ}$, respectively. This is not an artifact of the differential imaging because the two structures are also clearly visible on images where no differential imaging was applied. The structure and nature of this jet-like structure will be studied in more detail in a dedicated paper (Rigliaco et al., in prep.). A fainter counter-jet-like structure is also visible both in the IRDIS and IFS images at an angle of about $180^{\circ}$ with respect to the jet-like structure described above and it is identified with 5 in the IFS image in Fig. 3. Another curved structure is visible southeast of the star and apparently it starts from the base of the jet-like structure and not from the star itself. It is identified with 3 in the IFS image in Fig. 3 but it is even more clearly visible in Fig. 4. Its approximated position angle is $103.5^{\circ}$ and it could be part of an external disk of which could also be part the very faint structure identified 4 northwest of the star with a position angle of $305.9^{\circ} \pm 9.0^{\circ}$ at $\sim 180^{\circ}$ from the position of the companion (tagged 2 in Fig. 3).

\subsection{Results from SINFONI data}

The companion was also retrieved in the SINFONI data as shown in the median image of the final datacube obtained applying the ADI method that is shown in Fig. 5. From the final datacube, applying aperture photometry at each single wavelength image, we were then able to extract the spectrum for the companion that was then corrected for telluric absorption using data from a B2 standard star (HD 203617) observed during the same night and at a similar airmass. The extracted SINFONI spectrum is very noisy at shorter and longer wavelengths and, for this reason, the reduction of the telluric absorption was not effective at those wavelengths and we decided to retain only wavelengths between $1.50 \mu \mathrm{m}$ and $1.75 \mu \mathrm{m}$ remaining with 1282 wavelengths that were then used to perform the cross-correlation procedure described in Sect. 5.3.

\section{Discussion}

\subsection{Characterization of the companion through evolutionary models}

The very strong and variable absorption of the CrA region does not allow us to blindly use the determinations of the absorption listed in Sect. 3. To overcome this problem we exploited the isochrones of the BT-Settl models (Allard 2014). In Fig. 6 we show the 1 and $2 \mathrm{Myr}$ isochrones for $J$ versus $J-H$ compared with the value of $J-H=2.18 \pm 0.11$ (red vertical line) calculated for the companion of R CrA from the extracted apparent magnitudes that are listed in the second column of Table 3 . As a first step we calculated the value of the reddening $E(J-H)$ taking as reference the median value of $J-H$ for the $1 \mathrm{Myr}$ isochrone in the region with $6<J<9$ where the value of $J-H$ is almost constant. We then calculated the corresponding value of $A_{J}$ using the formula from Cardelli et al. (1989). With this value and applying the appropriate correction for the distance modulus we can retrieve the absolute magnitude in the $J$ band that, through the use of the BT-Settl models, allows us to estimate the mass of the companion. Using the BT-Settl models we can then obtain the absolute magnitude for the $H$ band as well and 
from this calculate an updated value for $J-H$ and for $E(J-H)$, comparing the latter to the original value for the companion. We then checked if the initial value of $E(J-H)$ corresponds to the new value that we obtain from the model. We iterated the steps described above until the difference between the input and output values for $E(J-H)$ is less than $0.01 \mathrm{mag}$. Once we obtained the final values for $A_{J}$ and for the absolute magnitude in the $J$ band, we also retrieved the same values for all the other spectral bands. These results are listed in column four and five of Table 3 .

Moreover, the absolute magnitudes listed in Table 3 allow us to define some of the main physical characteristics of R CrA B through the use of the BT-Settl models. We obtained values of $0.29 \pm 0.08 M_{\odot}, T_{\text {eff }}=3270 \pm 175 \mathrm{~K}, \log g=3.45 \pm 0.06$ and finally a radius of $16.7 \pm 4.2 R_{\mathrm{Jup}}$. To evaluate the errors in these results we took into account the uncertainties on the distance, age, and magnitudes of the host star. According to Pecaut \& Mamajek (2013) the value of $T_{\text {eff }}$ that we found would correspond to a spectral type of M3-M3.5. The relatively low surface gravity found for the companion, coupled with the large radius, are hints of a not fully completed gravitational collapse as expected for such young objects.

The value of $A_{J}$ was used to derive an estimate of $A_{V}=$ 15.89 and, assuming a value of $R_{V}=4.7$, we obtained a $E(B-V)=3.38$. These values were then used to correct the spectrum obtained with the procedure described in Sect. 4.1 using the IDL ccm_unred routine based on the Cardelli et al. (1989) formula. While the uncorrected spectrum is very red, after the correction we have a much bluer spectrum typical of a stellar object.

\subsection{Fitting with template spectra and atmospheric models}

To further characterize the companion we tried to fit its extracted spectrum with libraries of template spectra with the aim to define its spectral type. To this aim we used the library of field BDs spectra taken from the Spex Prism spectral Libraries ${ }^{2}$ (Burgasser 2014) and the library of spectra taken from Allers \& Liu (2013). The procedure is similar to those adopted in Mesa et al. (2016), Mesa et al. (2018), and Ligi et al. (2018). For the fit we did not use the three spectral points at shorter wavelengths that are anomalously high as can be seen in Fig. 7. This is probably due to a contamination arising from the particular configuration of the IFS raw data (see, e.g., Claudi et al. 2008). In these data the faint blue ends of the R CrA B spectrum in one spaxel are adjacent to the very bright red ends of the spectrum on a near spaxel. This results in some light from the bright red pixel leaking toward the faint blue pixels. The final results for the fit procedure are shown in Fig. 8 and are compatible with an early- $M$ spectral type for the companion.

We also tried to fit the extracted spectra with a set of BTSettl and BT-NextGen atmospheric models (Allard et al. 1997; Allard 2014) with a grid covering $T_{\text {eff }}$ between 900 and $4000 \mathrm{~K}$ with a step of $100 \mathrm{~K}$ and a $\log g$ ranging between 2.5 and 5.5 dex with a step of 0.5 . All the models were for a solar metallicity. The results of this procedure are shown in Fig. 9. From these results it is difficult to draw a conclusion given that a lot of different models fit well with our results. However, the results are compatible with a high $T_{\text {eff }}$ in the range between $3500 \mathrm{~K}$ and $4000 \mathrm{~K}$. Also, they seem to favor high surface gravity models. These results are in contrast with what we obtained in Sect. 5.1, where the value of $T_{\text {eff }}$ was slightly lower and moreover we favored a low surface gravity result.

\footnotetext{
2 http://pono.ucsd.edu/ adam/browndwarfs/spexprism/
}

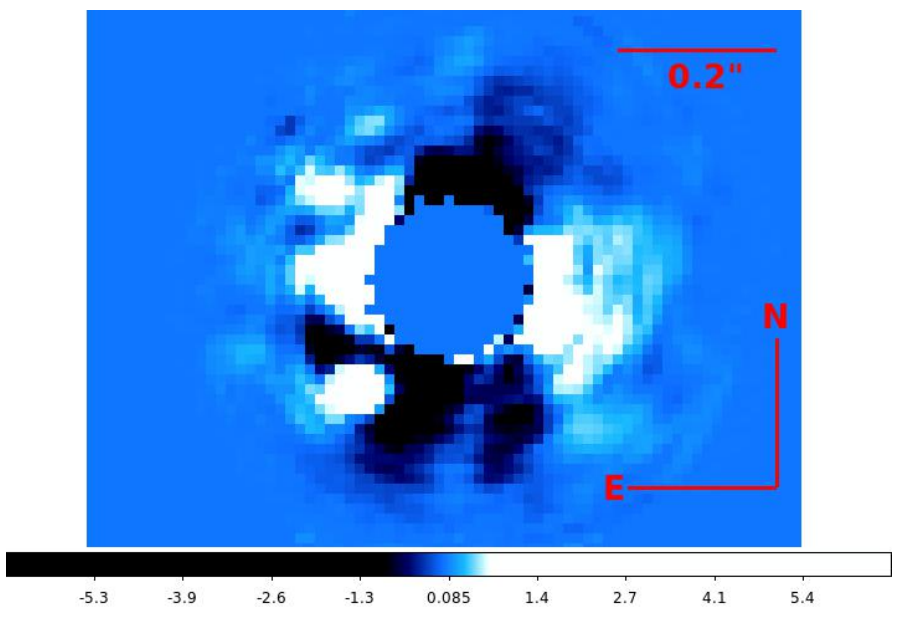

Fig. 5. R CrA image resulting from the median of the final SINFONI datacube obtained applying the ADI technique.

\subsection{Characterization of the companion through SINFONI data}

The spectrum extracted from the SINFONI data was used to obtain a further spectral classification of the object by comparing it with a library of template spectra of $K$ and $M$ stars taken from the IRTF library (Cushing et al. 2005; Rayner et al. 2009). The comparison was done through the spectral lines and, to avoid the final result to be influenced by the slope of the spectra strongly dependent on the very uncertain value of the extinction, we divided each spectrum that we used for a smoothed version of itself to eliminate any slope. The smoothing was done with the standard SMOOTH IDL procedure using a smoothing window of 100 . The spectra were then compared via the C_CORRELATE IDL routine trying to maximize the crosscorrelation index between them. Moreover, to explore the possible shift of the spectra due to the radial velocity of R CrA, this procedure was executed shifting the template spectra of arbitrary wavelength values. In any case, we always found that the highest cross-correlation indices were obtained for shifts very near to zero concluding that the radial velocity of $\mathrm{R} \mathrm{CrA}$ have to be very close to 0 . The same procedure was then repeated using a library of young stars spectra obtained by Manara et al. (2013) and Manara et al. (2017) from X-Shooter data. In Fig. 10 we show the values of the cross-correlation index as a function of the spectral type that we obtain from the procedure described above showing, in orange, the values obtained from the IRTF data and, in green, the values obtained from the X-Shooter data. The evolution of the cross-correlation index is very similar in both cases. The maximum values are obtained for spectral types between M0 and M1.5, where the value of the index is almost stable. On the low mass side the values of the indices diminish with a steep slope, while a shallower slope is found on the side of the $K$ stars. In Fig. 11 we represent the normalized spectrum of $\mathrm{R} \mathrm{CrA} \mathrm{B} \mathrm{together} \mathrm{with} \mathrm{those} \mathrm{of} \mathrm{some} \mathrm{of} \mathrm{the} \mathrm{best} \mathrm{fit} \mathrm{spectra} \mathrm{from}$ the IRTF library. The fit of the spectral lines between the compared spectra is good with values of the cross-correlation index between 0.46 and 0.48 for the best fit spectra. In the image we denoted some of the most prominent lines in the spectra identifying lines from $\mathrm{Mg}$ I, Fe I, Si I, and Al I. An evident line in the R CrA B spectrum is also present at $1.67 \mu \mathrm{m}$ but it is not present in the template spectra. This is probably a telluric line that has not been completely deleted by the subtraction of the telluric spectrum standard and for this reason we marked it with 
Table 2. Astrometric results obtained for $\mathrm{R} \mathrm{CrA} \mathrm{B}$.

\begin{tabular}{lcccc}
\hline \hline Date & $\Delta$ RA $\left({ }^{\prime \prime}\right)$ & $\Delta \operatorname{Dec}\left({ }^{\prime \prime}\right)$ & $\rho\left({ }^{\prime \prime}\right)$ & PA \\
\hline $2015-06-10$ & $0.098 \pm 0.004$ & $-0.121 \pm 0.004$ & $0.156 \pm 0.004$ & $141.0 \pm 0.2$ \\
$2016-08-10$ & $0.117 \pm 0.004$ & $-0.140 \pm 0.004$ & $0.182 \pm 0.004$ & $140.0 \pm 0.2$ \\
$2018-06-19$ & $0.134 \pm 0.001$ & $-0.129 \pm 0.001$ & $0.184 \pm 0.001$ & $134.2 \pm 0.2$ \\
$2018-08-16$ & $0.132 \pm 0.004$ & $-0.128 \pm 0.004$ & $0.184 \pm 0.004$ & $134.0 \pm 0.2$ \\
\hline
\end{tabular}

Table 3. Photometric results obtained for R CrA B.

\begin{tabular}{lcccc}
\hline \hline Spectral band & App. mag & $\Delta$ Mag & $A_{\lambda}$ & Dered abs. mag. \\
\hline$Y$ & $16.45 \pm 0.05$ & $5.53 \pm 0.19$ & $5.83 \pm 0.32$ & $4.70 \pm 0.27$ \\
$J$ & $14.60 \pm 0.08$ & $6.24 \pm 0.74$ & $4.48 \pm 0.26$ & $4.20 \pm 0.26$ \\
$H$ & $12.42 \pm 0.03$ & $5.47 \pm 0.32$ & $3.01 \pm 0.38$ & $3.49 \pm 0.35$ \\
$K 1$ & $10.63 \pm 0.01$ & $6.00 \pm 0.33$ & $1.36 \pm 0.30$ & $3.35 \pm 0.29$ \\
$K 2$ & $10.27 \pm 0.01$ & $5.64 \pm 0.33$ & $1.18 \pm 0.28$ & $3.17 \pm 0.27$ \\
\hline
\end{tabular}

Notes. The second column lists its apparent magnitudes before the correction for the extinction, the third column the differences in magnitudes with respect to the central star, the fourth column lists the values of the estimated extinction, and finally the fifth column presents its absolute magnitudes after the correction for the extinction.

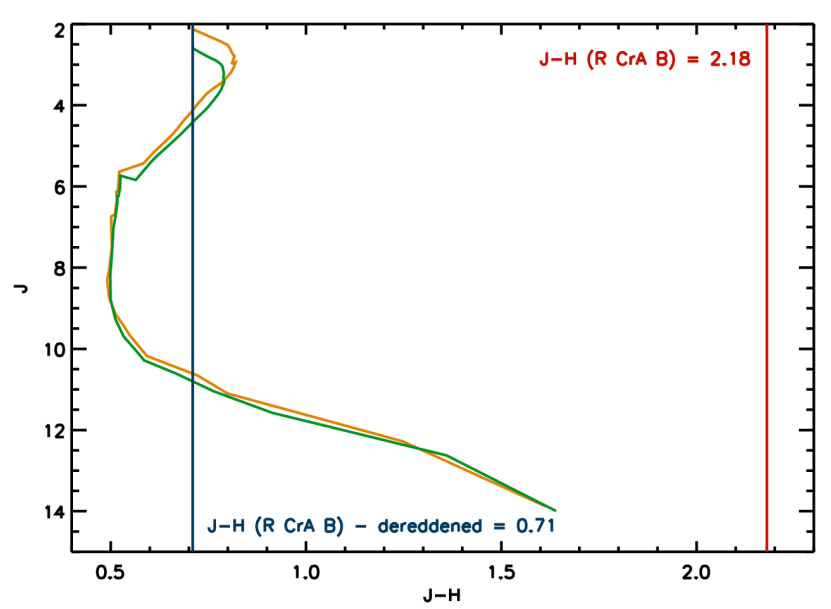

Fig. 6. Isochrones $J$ vs. $J-H$ in the case of $1 \mathrm{Myr}$ (orange line) and $2 \mathrm{Myr}$ (green line). The red vertical line represents the $J-H$ value calculated for R CrA B.

" $T$ " in Fig. 11. Using the spectral classification obtained from this method, we obtained for R CrA B a $T_{\text {eff }}$ between 3650 and $3870 \mathrm{~K}$ that, from the isochrones for pre-main sequence stars by Baraffe et al. (2015), corresponds to a mass between 0.47 and $0.55 M_{\odot}$.

We also used the $\mathrm{H}$ lines in the spectral region observed with SINFONI to search for any evidence of accretion. However, the upper limit to the equivalent width (EW), obtained from the median of $\mathrm{H}$ lines in this region, is of $0.05 \mathrm{~nm}$. As a comparison we can consider the Orion low mass stars considered in Rigliaco et al. (2012) that have an EW of $\sim 0.1 \mathrm{~nm}$. We can then conclude that there is no evidence of accretion for $\mathrm{R} \mathrm{CrA} \mathrm{B} \mathrm{even}$ if we have to consider that accretion for this object is very probably variable while we obtained a spectrum in just one epoch.

\subsection{Orbital parameters}

Using the astrometric data listed in Table 2 we performed a Monte Carlo simulation to constrain the orbital parameters using the Thiele-Innes formalism (Binnendijk 1960) as described

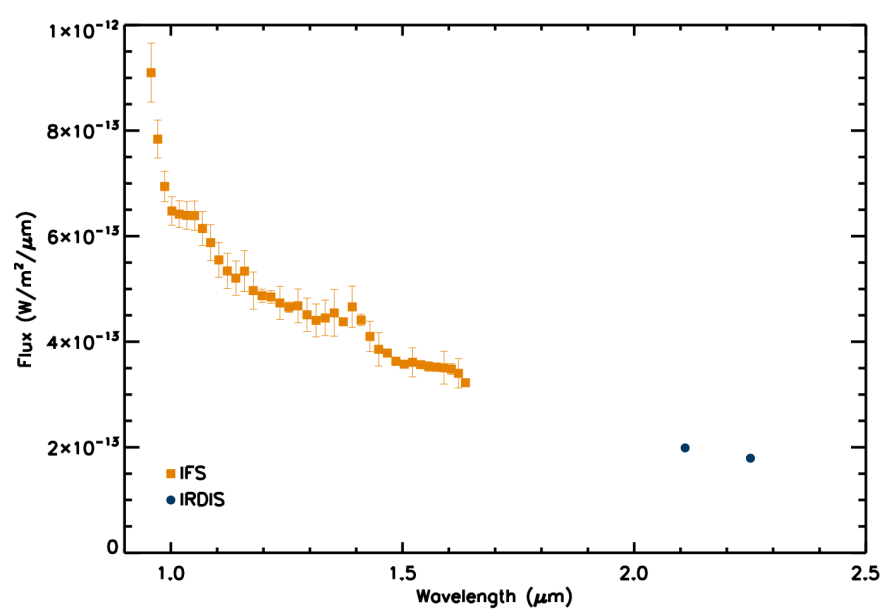

Fig. 7. Final spectrum extracted for R CrA B from SPHERE data after applying the correction for the extinction. The orange squares are measures obtained with IFS while the blue circles are obtained with IRDIS. In this last case the error bars are smaller than the symbol size.

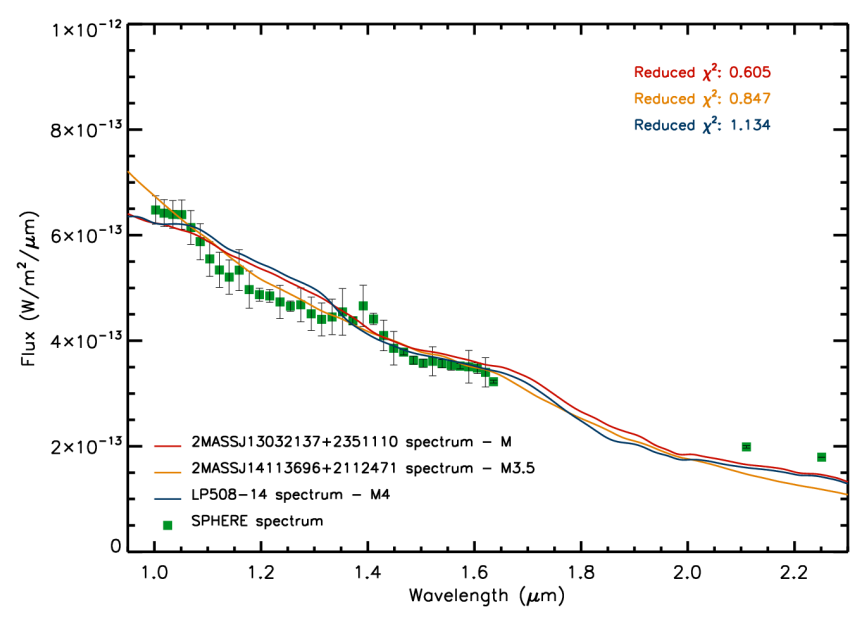

Fig. 8. Comparison of the R CrA B spectrum obtained from SPHERE (green squares) with three of the best fit template spectra. 


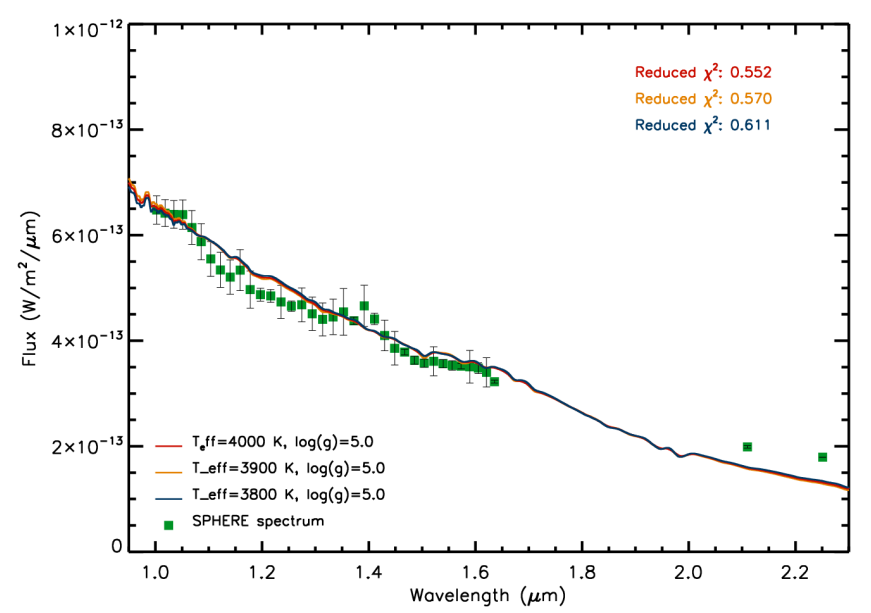

Fig. 9. Comparison of the R CrA B spectrum obtained from SPHERE (green squares) with three of the best fit BT-Settl atmospheric models.

in Desidera et al. (2011), Zurlo et al. (2013), and Zurlo et al. (2018) and adopting the convention by Heintz (2000). The simulation generates by $5 \times 10^{7}$ random orbital elements and rejects all the central orbits that do not fit the astrometric data. Given that the mass of the star is poorly constrained through previous studies, we also decided to include it between the varying parameters of our simulation considering a very large mass range from $2 M_{\odot}$ to $30 M_{\odot}$. We also assumed a large variation of the mass of the companion between 1 and $1000 M_{\text {Jup }}$. The simulation found 144249 orbits consistent with the observational data. The results of this procedure for the main parameters are shown in Fig. 12. The simulation clearly prefers a low mass for the whole system, an eccentricity of $\sim 0.4$, a semimajor axis of $27-28$ au, and an inclination of $\sim 70^{\circ}$. However, we are not able to obtain a good value for the companion mass given that its distribution is very uniform and for the period even if in this case periods of less than $30 \mathrm{yr}$ are clearly excluded and we have a very smoothed peak at about $65-70 \mathrm{yr}$. The availability of long-term radial velocity data could help constrain the system mass ratio. Unfortunately, we did not find any data of this type for R CrA.

\subsection{Mass limits around R CrA}

With the aim to estimate the mass limits for other possible objects around R CrA we calculated the brightness contrast following the method devised in Mesa et al. (2015). In this case, however, to overcome the problems arising from the variability of R CrA, we used as reference the star HIP 63847 following the same procedure used to obtain the results in Sect. 4.1. We then obtained the contrast in absolute magnitude and corrected it for the extinction that we calculated for the companion in Table 3. While these values are not necessarily valid for the entire region around $\mathrm{R} C r A$, we used them to give an estimate of the effective contrast. This result was then used in association with the AMES-Dusty models to evaluate the mass limits for other objects around the star. The results of this procedure are shown in Fig. 13. Because of the much larger extinction at shorter wavelengths, IFS is in this case less effective in finding low mass companions, hence we can use IRDIS to set these limits. At separations from the star less than $100 \mathrm{au}$, we are able to obtain mass limits variable, according to the separation, between 6 and $2.5 M_{\text {Jup }}$ while at larger separations we can reach an almost stable mass limit on the order of $1.5 \mathrm{M}_{\mathrm{Jup}}$.

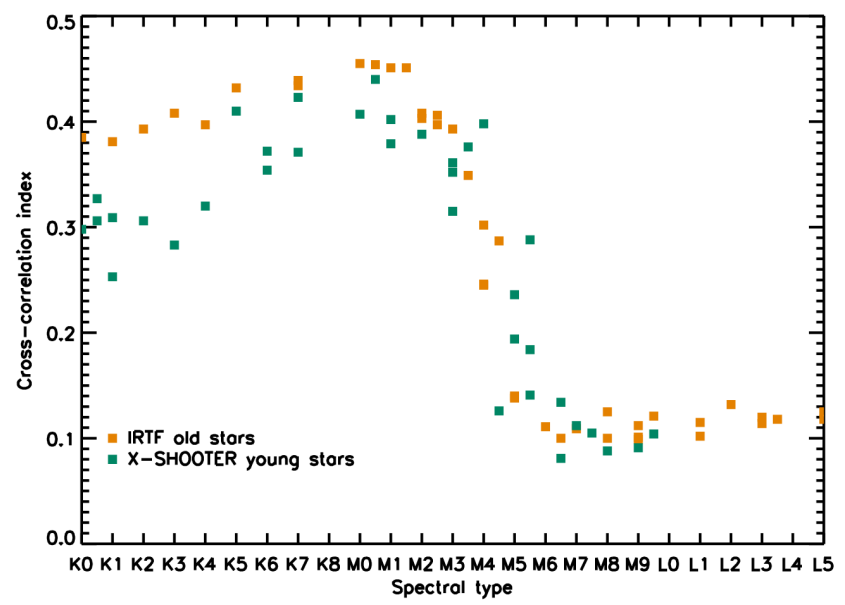

Fig. 10. Values of the cross-correlation index between the SINFONI spectrum of RCrAB and template spectra from the IRTF library (orange squares) and X-Shooter young stars (green squares) vs. the spectral type of the template spectra.

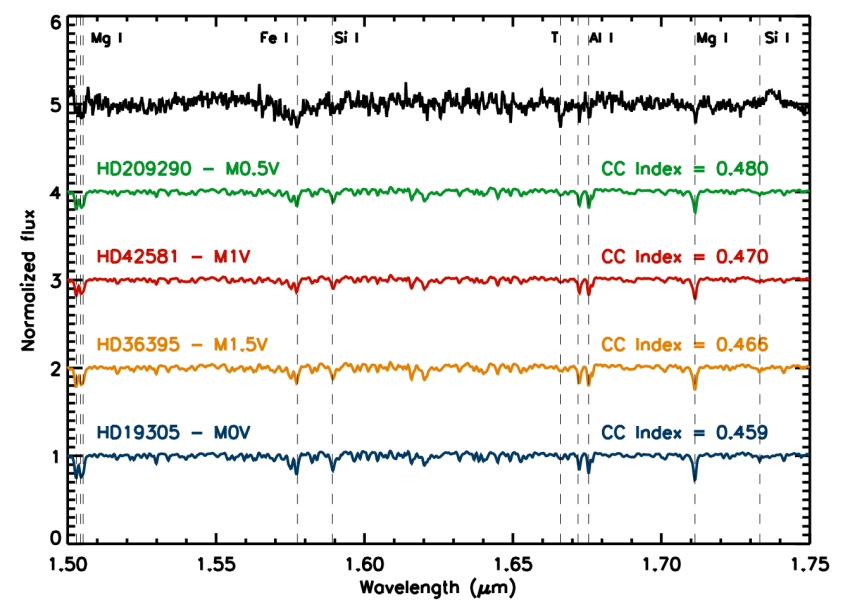

Fig. 11. Comparison of the R CrA B (black line) spectrum with those of some of the best fit spectra from the spectral libraries.

\section{Conclusions}

In this paper we report the results from the SPHERE multiepoch observations of the R CrA system. The central star is a very young $(\sim 1 \mathrm{Myr}) \mathrm{HAeBe}$ object with a strong reddening due to the presence of circumstellar material. The star is strongly variable on period of $\sim 66$ days and, moreover, it has strong differences in magnitude between different spectral bands. These facts make a proper photometric characterization of companion objects difficult.

A companion was found around the star and, thanks to the astrometric measurements taken during the four observing epochs, we were able to confirm that it is gravitationally bound to the star. Also, we were able to extract a spectrum of the companion. After correction for extinction in the direction of the companion and through the comparison with the BT-Settl evolutionary models we were able to infer for the companion a mass of $0.29 \pm 0.08 M_{\odot}$. Hence, the companion is an early- $M$ spectral type star deeply embedded in its dust envelope. This case is then very similar to that of the two companions of T Tau, T Tau S a, and T Tau S b (Dyck et al. 1982; Kasper et al. 2016). An alternative explanation of the strong extinction in the direction of the companion is that it could be due to the presence 

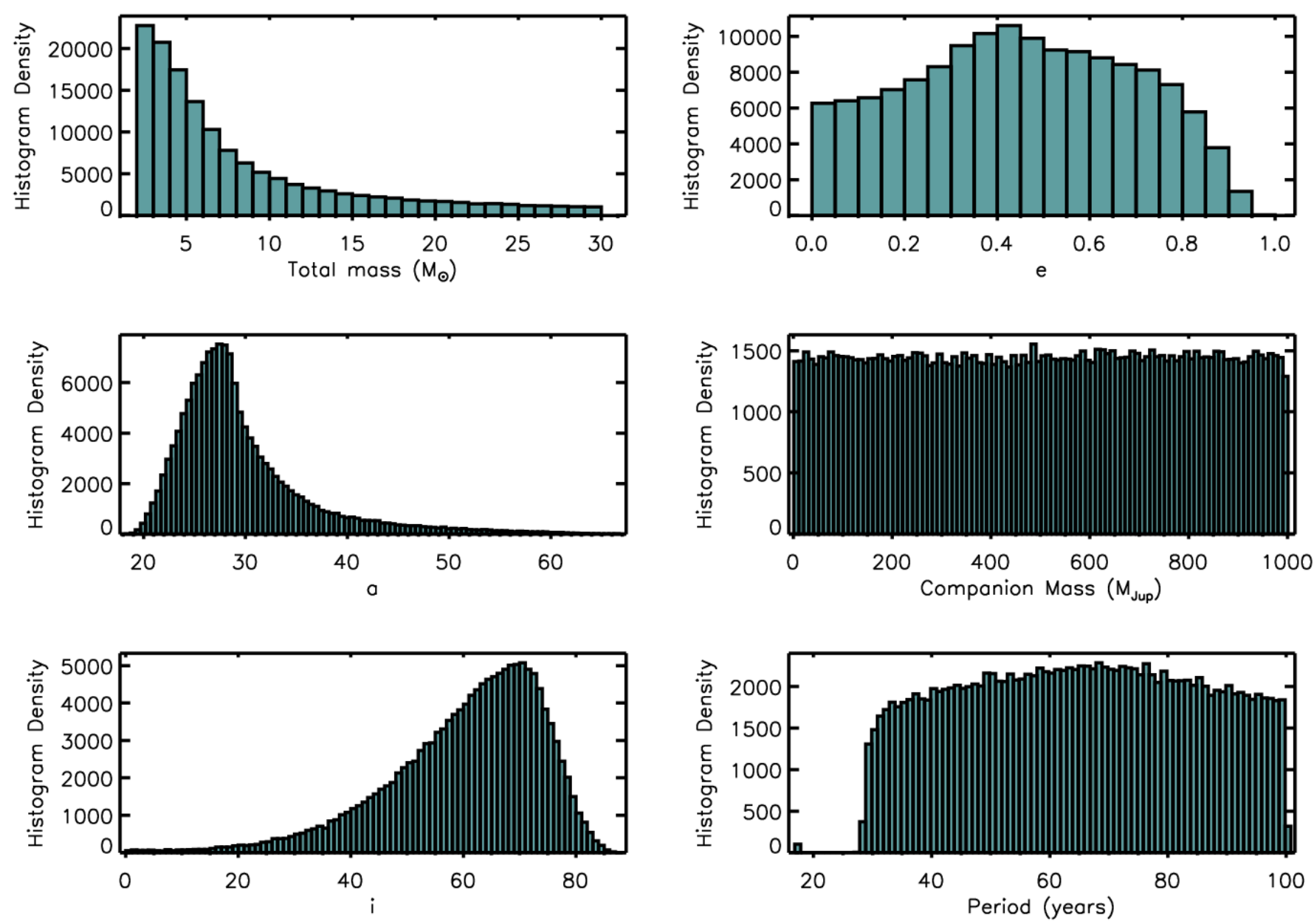

Fig. 12. Distribution histograms obtained from the Monte Carlo simulation described in Sect. 5.4 for the total mass of the system in solar masses (upper left panel), ellipticity (upper right panel), the semimajor axis in au (center left panel), companion mass in $M_{\mathrm{Jup}}$ (center right panel), inclination of the orbit (bottom left panel), and period in years (bottom right panel).

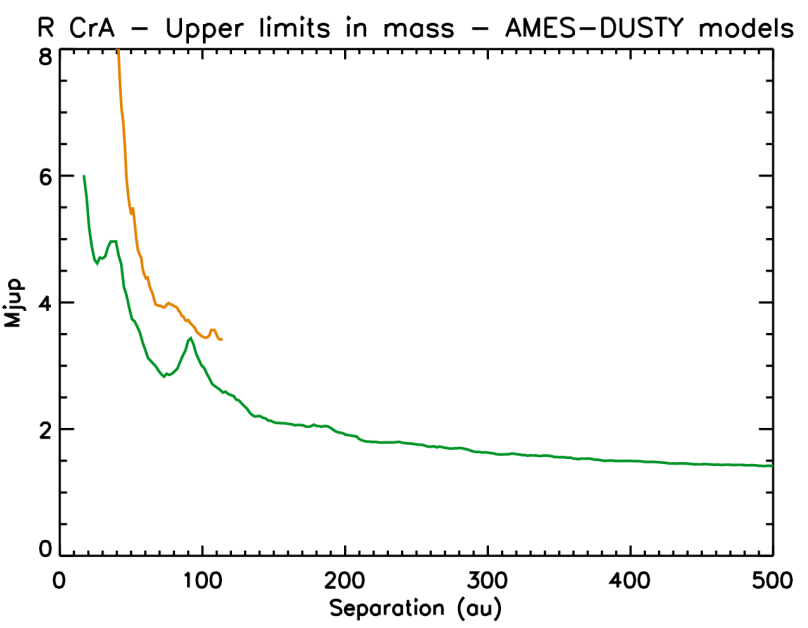

Fig. 13. Mass limits expressed in $M_{\mathrm{Jup}}$ vs. the separation expressed in au for possible objects around R CrA. The IFS limits are given by the orange line while the IRDIS limits are given by the green line.

of an external disk seen at very large inclination. Even though we found some hints of the presence of this external disk, we are not able at the moment to disentangle between the two explanations of the large extinction in the direction of the companion. The spectral classification of the companion is further confirmed comparing template spectra to the companion spectrum obtained using SINFONI. The results obtained with the latter instrument provide a slightly higher value both for the mass $\left(0.47-0.55 M_{\odot}\right)$ and for $T_{\text {eff }}(3650-3870 \mathrm{~K})$ than what obtained from the SPHERE data. This discrepancy could be due to the fact that the extinction value determined from SPHERE data is slightly underestimated, obtaining in this latter case a value of $A_{J}=5.00 \mathrm{mag}$. Moreover, given that the classification through the SINFONI data relies on spectral lines that are not affected by the extinction, we believe that the latter are a more reliable estimate of the companion mass. The higher temperature derived from the spectral lines through SINFONI data might be due to the presence of a hotter region on the stellar surface related to accretion. In addition, we notice that no emission line was detected in our SINFONI spectrum, although several lines in the Brackett series are included in the observed spectral range with an upper limit to the EW of $0.05 \mathrm{~nm}$. It is then worth noticing that, using for the star the distance of $94.9 \mathrm{pc}$ derived from the Gaia parallax, we would obtain from the comparison with the BT-Settl evolutionary models a mass on the order of $0.14 \pm 0.03 M_{\odot}$ corresponding to a spectral type of M5-M6. This would be even more in contrast with the mass determination obtained from the SINFONI spectrum of the companion that is not depending from the distance of the star. This gives even more strength to our estimate of the distance of the system made in Sect. 3. 
The position of the stellar companion probably in a gap in the disk around the primary star of the system could make this object a candidate for formation through disk instability as proposed, for example, by Forgan et al. (2016) for massive young protostars with a large disk-star mass ratio. A similar object has recently been discovered through ALMA observations around the young stellar object G11.92-0.61 MM1 (Ilee et al. 2018). In any case, to confirm this possibility we will need more precise information both about the physical characteristics (e.g., its mass that is still poorly defined) of the primary star of the system and on the effective location of the companion into the gap of the disk for which we have at the moment just non-conclusive hints.

Aside from the presence of the companion, the environment around $\mathrm{R} \mathrm{CrA}$ is further enriched by the presence of a number of extended structures. The most notable of these is surely the bright jet-like structure northeast of the star. Our images highlight moreover that this jet-like structure is itself formed by at least two substructures. A less bright counter-jet-like structure on the opposite side of the star is also visible together with some arc-like structures that could be part of an external disk. Instead, in our images it is not possible to image the inner disk that the star is known to host. We will discuss the nature and the origin of the jet-like structure and of all the other extended structures in this system in a following dedicated paper.

Acknowledgements. The authors thank the anonymous referee for the constructive comments that helped to strongly improve the quality of the present work. This work has made use of the SPHERE Data Center, jointly operated by OSUG/IPAG (Grenoble), PYTHEAS/LAM/CeSAM (Marseille), OCA/Lagrange (Nice) and Observatoire de Paris/LESIA (Paris). This work has made use of data from the European Space Agency (ESA) mission Gaia (https://www . cosmos.esa.int/gaia), processed by the Gaia Data Processing and Analysis Consortium (DPAC, https://www.cosmos.esa.int/web/gaia/dpac/ consortium). Funding for the DPAC has been provided by national institutions, in particular the institutions participating in the Gaia Multilateral Agreement. This research has made use of the SIMBAD database, operated at CDS, Strasbourg, France. D.M. acknowledges support from the ESO-Government of Chile Joint Comittee program "Direct imaging and characterization of exoplanets”. D.M., A.Z., V.D.O., R.G., R.U.C., S.D., C.L. acknowledge support from the "Progetti Premiali" funding scheme of the Italian Ministry of Education, University, and Research. A.Z. acknowledges support from the CONICYT + PAI/ Convocatoria nacional subvención a la instalación en la academia, convocatoria 2017 + Folio PAI77170087. M.G.U.G., G.L. and S.A. acknowledge support from the project PRIN-INAF 2016 The Cradle of Life - GENESISSKA (General Conditions in Early Planetary Systems for the rise of life with SKA). GL and MGUG have received funding from the European Union's Horizon 2020 research and innovation programme under the Marie SklodowskaCurie grant agreement No. 823823. G.P. and M.L. acknowledge financial support from the ANR of France under contract number ANR-16-CE31-0013 (PlanetForming-Disks). D.F. acknowledges financial support provided by the Italian Ministry of Education, Universities and Research, project SIR (RBSI14ZRHR). R.L. has received funding from the European Union's Horizon 2020 research and innovation program under the Marie Sklodowska-Curie grant agreement No. 664931. S.A. acknowledges the support by INAF/Frontiera through the "Progetti Premiali" funding scheme of the Italian Ministry of Education, University, and Research. This publication makes use of VOSA, developed under the Spanish Virtual Observatory project supported from the Spanish MINECO through grant AyA2017-84089. This work has been supported by a grant from the Agence Nationale de la Recherche (grant ANR-14-CE33-0018). SPHERE is an instrument designed and built by a consortium consisting of IPAG (Grenoble, France), MPIA (Heidelberg, Germany), LAM (Marseille, France), LESIA (Paris, France), Laboratoire Lagrange (Nice, France), INAF-Osservatorio di Padova (Italy), Observatoire de Genève (Switzerland), ETH Zurich (Switzerland), NOVA (Netherlands), ONERA (France) and ASTRON (Netherlands), in collaboration with ESO. SPHERE was funded by ESO, with additiona contributions from CNRS (France), MPIA (Germany), INAF (Italy), FINES (Switzerland) and NOVA (Netherlands). SPHERE also received funding from the European Commission Sixth and Seventh Framework Programmes as part of the Optical Infrared Coordination Network for Astronomy (OPTICON) under grant number RII3-Ct-2004-001566 for FP6 (2004-2008), grant number 226604 for FP7 (2009-2012) and grant number 312430 for FP7 (2013-2016).

\section{References}

Allard, F. 2014, in Exploring the Formation and Evolution of Planetary Systems, eds. M. Booth, B. C. Matthews, \& J. R. Graham, IAU Symp., 299, 271

Allard, F., Hauschildt, P. H., Alexander, D. R., \& Starrfield, S. 1997, ARA\&A, 35,137

Allard, F., Homeier, D., \& Freytag, B. 2012, Philos. Trans. R. Soc. London Ser. A, 370, 2765

Allers, K. N., \& Liu, M. C. 2013, ApJ, 772, 79

Baraffe, I., Homeier, D., Allard, F., \& Chabrier, G. 2015, ApJ, 577, A42

Bayo, A., Rodrigo, C., Barrado, Y., et al. 2008, ApJ, 492, 277

Beuzit, J.-L., Vigan, A., Mouillet, D., et al. 2019, A\&A, submitted [arXiv:1902 . 04080]

Bibo, E. A., The, P. S., \& Dawanas, D. N. 1992, ApJ, 260, 293

Binnendijk, L. 1960, Properties of Double Stars; A Survey of Parallaxes and Orbits (Philadelphia, PA: University of Pennsylvania Press)

Bonnet, H., Conzelmann, R., Delabre, B., et al. 2004, in Advancements in Adaptive Optics, eds. D. Bonaccini Calia, B. L. Ellerbroek, \& R. Ragazzoni, Proc. SPIE, 5490, 130

Bowler, B. P. 2016, PASP, 128, 102001

Burgasser, A. J. 2014, Astron. Soc. India Conf. Ser., 11

Cardelli, J. A., Clayton, G. C., \& Mathis, J. S. 1989, ApJ, 345, 245

Chauvin, G., Desidera, S., Lagrange, A. M., et al. 2017, in SF2A-2017: Proceedings of the Annual meeting of the French Society of Astronomy and Astrophysics, eds. C. Reylé, P. Di Matteo, F. Herpin, et al., 331

Chen, H., Grenfell, T. G., Myers, P. C., \& Hughes, J. D. 1997, ApJ, 478, 295

Chen, C. H., Pecaut, M., Mamajek, E. E., Su, K. Y. L., \& Bitner, M. 2012, ApJ, 756,133

Clark, S., McCall, A., Chrysostomou, A., et al. 2000, MNRAS, 319, 337

Claudi, R. U., Turatto, M., Gratton, R. G., et al. 2008, SPIE Conf. Ser., 7014, 70143E

Cushing, M. C., Rayner, J. T., \& Vacca, W. D. 2005, ApJ, 623, 1115

Cutri, R. M., Skrutskie, M. F., van Dyk, S., et al. 2003, VizieR Online Data Catalog: II/246

de Zeeuw, P. T., Hoogerwerf, R., de Bruijne, J. H. J., Brown, A. G. A., \& Blaauw, A. 1999, AJ, 117, 354

Delorme, P., Meunier, N., Albert, D., et al. 2017, in SF2A-2017: Proceedings of the Annual meeting of the French Society of Astronomy and Astrophysics, eds. C. Reylé, P. Di Matteo, F. Herpin, et al., 347

Desidera, S., Carolo, E., Gratton, R., et al. 2011, ApJ, 533, A90

Dohlen, K., Langlois, M., Saisse, M., et al. 2008, SPIE Conf. Ser., 7014, 70143L

Ducati, J. R. 2002, VizieR Online Data Catalog: II/237

Dyck, H. M., Simon, T., \& Zuckerman, B. 1982, ApJ, 255, L103

Dzib, S. A., Loinard, L., Ortiz-León, G. N., Rodríguez, L. F., \& Galli, P. A. B. 2018, ApJ, 867, 151

Eisenhauer, F., Abuter, R., Bickert, K., et al. 2003, in Instrument Design and Performance for Optical/Infrared Ground-based Telescopes, eds. M. Iye, \& A. F. M. Moorwood, Proc. SPIE, 4841, 1548

Forbrich, J., Preibisch, T., \& Menten, K. M. 2006, ApJ, 446, 155

Forgan, D. H., Ilee, J. D., Cyganowski, C. J., Brogan, C. L., \& Hunter, T. R. 2016, MNRAS, 463, 957

Gaia Collaboration. 2018, VizieR Online Data Catalog: I/345

Galicher, R., Boccaletti, A., Mesa, D., et al. 2018, ApJ, 615, A92

Garcia Lopez, R., Natta, A., Testi, L., \& Habart, E. 2006, ApJ, 459, 837

Graham, J. A. 1992, in Star Formation in the Corona Australis Region, ed. B. Reipurth, 185

Graham, J. A. 1993, PASP, 105, 561

Gray, R. O., Corbally, C. J., Garrison, R. F., et al. 2006, AJ, 132, 161

Heintz, W. 2000, in Visual Binary Stars, ed. P. Murdin, 2855

Herbig, G. H. 1960, ApJS, 4, 337

Herbst, W., \& Shevchenko, V. S. 1999, AJ, 118, 1043

Hillenbrand, L. A., Strom, S. E., Vrba, F. J., \& Keene, J. 1992, ApJ, 397, 613

Hoeijmakers, H. J., Schwarz, H., Snellen, I. A. G., et al. 2018, ApJ, 617, A144

Ilee, J. D., Cyganowski, C. J., Brogan, C. L., et al. 2018, ApJ, 869, L24

Johnson, J. A., Aller, K. M., Howard, A. W., \& Crepp, J. R. 2010, PASP, 122, 905

Kasper, M., Santhakumari, K. K. R., Herbst, T. M., \& Köhler, R. 2016, ApJ, 593, A50

Koen, C., Kilkenny, D., van Wyk, F., \& Marang, F. 2010, MNRAS, 403, 1949

Kraus, S., Hofmann, K.-H., Malbet, F., et al. 2009, ApJ, 508, 787

Langlois, M., Vigan, A., Moutou, C., et al. 2013, in Proceedings of the Third AO4ELT Conference, eds. S. Esposito, \& L. Fini, 63

Lazareff, B., Berger, J.-P., Kluska, J., et al. 2017, ApJ, 599, A85

Ligi, R., Demangeon, O., Barros, S., et al. 2018, AJ, 156, 182

Malfait, K., Bogaert, E., \& Waelkens, C. 1998, ApJ, 331, 211

Manara, C. F., Testi, L., Rigliaco, E., et al. 2013, ApJ, 551, A107

Manara, C. F., Frasca, A., Alcalá, J. M., et al. 2017, ApJ, 605, A86 
Marois, C., Correia, C., Galicher, R., et al. 2014, in Adaptive Optics Systems IV, Proc. SPIE, 9148, 91480U

Marois, C., Lafrenière, D., Doyon, R., Macintosh, B., \& Nadeau, D. 2006a, ApJ, 641,556

Marois, C., Lafrenière, D., Macintosh, B., \& Doyon, R. 2006b, ApJ, 647, 612

Marshall, J. P., Moro-Martín, A., Eiroa, C., et al. 2014, ApJ, 565, A15

Mesa, D., Gratton, R., Zurlo, A., et al. 2015, ApJ, 576, A121

Mesa, D., Vigan, A., D’Orazi, V., et al. 2016, ApJ, 593, A119

Mesa, D., Baudino, J.-L., Charnay, B., et al. 2018, ApJ, 612, A92

Meshkat, T., Bonnefoy, M., Mamajek, E. E., et al. 2015, MNRAS, 453, 2378

Meyer, M. R., \& Wilking, B. A. 2009, PASP, 121, 350

Natta, A., Palla, F., Butner, H. M., Evans, II., N. J., \& Harvey, P. M. 1993, ApJ 406, 674

Neuhäuser, R., \& Forbrich, J. 2008, in The Corona Australis Star Forming Region, ed. B. Reipurth, 735

Pavlov, A., Möller-Nilsson, O., Feldt, M., et al. 2008, SPIE Conf. Ser., 7019, 39

Pecaut, M. J., \& Mamajek, E. E. 2013, ApJS, 208, 9

Percy, J. R., Grynko, S., Seneviratne, R., \& Herbst, W. 2010, PASP, 122, 753

Racine, R., Walker, G. A. H., Nadeau, D., Doyon, R., \& Marois, C. 1999, PASP, 111,587

Rayner, J. T., Cushing, M. C., \& Vacca, W. D. 2009, ApJS, 185, 289

Rigliaco, E., Natta, A., Testi, L., et al. 2012, ApJ, 548, A56

Sicilia-Aguilar, A., Henning, T., Kainulainen, J., \& Roccatagliata, V. 2011, ApJ 736,137

Sivaramakrishnan, A., \& Oppenheimer, B. R. 2006, ApJ, 647, 620

Soummer, R., Pueyo, L., \& Larkin, J. 2012, ApJ, 755, L28

Takami, M., Bailey, J., \& Chrysostomou, A. 2003, ApJ, 397, 675

Taylor, K. N. R., \& Storey, J. W. V. 1984, MNRAS, 209, 5P

The, P. S. 1994, in The Nature and Evolutionary Status of Herbig Ae/Be Stars, eds. P. S. The, M. R. Perez, \& E. P. J. van den Heuvel, ASP Conf. Ser., 62, 23

Vigan, A., Moutou, C., Langlois, M., et al. 2010, MNRAS, 407, 71

Ward-Thompson, D., Warren-Smith, R. F., Scarrott, S. M., \& Wolstencroft, R. D. 1985, MNRAS, 215, 537

Wilking, B. A., McCaughrean, M. J., Burton, M. G., et al. 1997, AJ, 114, 2029

Zechmeister, M., \& Kürster, M. 2009, ApJ, 496, 577

Zurlo, A., Vigan, A., Hagelberg, J., et al. 2013, ApJ, 554, A21

Zurlo, A., Vigan, A., Mesa, D., et al. 2014, ApJ, 572, A85

Zurlo, A., Mesa, D., Desidera, S., et al. 2018, MNRAS, 480, 35

INAF-Osservatorio Astronomico di $\quad$ Padova,
dell'Osservatorio 5, 35122 Padova, Italy
e-mail: dino.mesa@inaf.it

2 INCT, Universidad De Atacama, Calle Copayapu 485, Copiapó, Atacama, Chile
3 Univ. Grenoble Alpes, CNRS, IPAG, 38000 Grenoble, France

4 Nucleo de Astronomia, Facultad de Ingenieria y Ciencias, Universidad Diego Portales, Av. Ejercito 441, Santiago, Chile

5 Escuela de Ingenieria Industrial, Facultad de Ingenieria y Ciencias, Universidad Diego Portales, Av. Ejercito 441, Santiago, Chile

6 Aix Marseille Univ., CNRS, CNES, LAM, Marseille, France

7 Hamburger Sternwarte, Gojenbergsweg 112, 21029 Hamburg, Germany

8 LESIA, Observatoire de Paris, PSL Research University, CNRS, Sorbonne Universités, UPMC Univ. Paris 06, Univ. Paris Diderot, Sorbonne, Paris Cité, 5 Place Jules Janssen, 92195 Meudon, France

9 Univ. Lyon, Univ. Lyon 1, ENS de Lyon, CNRS, CRAL UMR 5574, 69230 Saint-Genis-Laval, France

10 Dipartimento di Fisica, Universitá Degli Studi di Milano, Via Celoria, 16, 20133 Milano, Italy

11 INAF-Osservatorio Astronomico di Roma, Via di Frascati 33, 00078 Monte Porzio Catone, Italy

12 Unidad Mixta Internacional Franco-Chilena de Astronoma (CNRS, UMI 3386), Departamento de Astronoma, Universidad de Chile, Camino El Observatorio 1515, Las Condes, Santiago, Chile

13 INAF-Osservatorio Astrofisico di Arcetri, Largo E. Fermi 5, 50125 Firenze, Italy

14 Max-Planck-Institut für Astronomie, Königstuhl 17, 69117 Heidelberg, Germany

15 European Southern Observatory (ESO), Karl-Schwarzschild-Str. 2 , 85748 Garching bei München, Germany

16 Department of Astronomy, University of Michigan, 1085 S. University Ave, Ann Arbor, MI 48109-1107, USA

17 Space Sciences, Technologies \& Astrophysics Research (STAR) Institute, Université de Liege, Allée du Six Aout 19c, 4000 Sart Tilman, Belgium

18 Geneva Observatory, University of Geneva, Chemin des Maillettes 51, 1290 Versoix, Switzerland

19 AlbaNova University Center, Stockholm University, Stockholm, Sweden

20 INAF-Osservatorio Astronomico di Brera, Via E. Bianchi 46, 23807 Merate, Italy

21 DOTA, ONERA, Université Paris Saclay, 91123 Palaiseau, France

22 Anton Pannekoek Institute for Astronomy, Science Park 904, 1098 XH Amsterdam, The Netherlands 